\title{
A review of the recent investigations regarding texturized cutting tools
}

\author{
Przegląd najnowszych badań \\ dotyczących strukturyzowanych narzędzi do cięcia
}

This paper presents some important, recently performed investigations on the laser texturing technology applied to the PCD and PCBN cutting tools and some resulting process outputs including cutting forces, tool wear indexes concerning both rake and flank tool faces. It was documented that the properly texturized tool faces results in a substantial reduction of cutting forces, elimination of the adhesion interaction between the tool and the chip, and reduction of abrasive and diffusion tool wear. The role of additional lubrication supply to the cutting zone with modified contact properties is discussed.

KEYWORDS: tool texturing, laser technique, cutting forces, tool wear

\section{Introduction}

Micro-texturing is applied to cutting tool surfaces for a number of reasons, including alteration of adhesion properties (mainly to reduce workpiece material adhesion/build-up edge (BUE) formation), enhancing cutting zone lubrication under different cooling strategies and preventing accelerated tool wear $[8,9]$. Texturing is applied mainly to rake faces of the cutting inserts, but it can also be applied to the flank face, for example to mitigate flank wear. Textures are usually applied in the immediate vicinity of the tool cutting edge and can take the form of grooves of varying orientation in respect to the main cutting edge, dimples, pits or convex geometries of various shapes. Selected examples of surface texturing designs are shown in Fig. 1. Naturally, the impact of tool texturing on numerous aspects of cutting process performance will vary depending on applied patterns.

Depending on the application and desired effects, parameters of surface texturing geometry - such as

a)

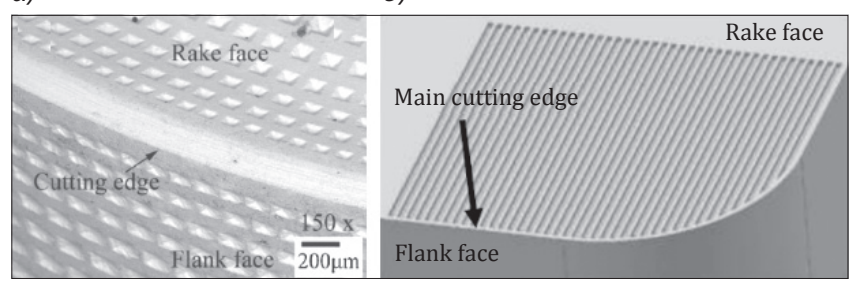

Fig. 1. Tool surface texturing in the form of concave pyramidical pits (a) and grooves parallel to the main cutting edge $(b)[1,2]$ depth, width/diameter and spacing - may vary. When using laser processing, obtaining these structures necessitates the appropriate adjustment of laser processing parameters. The matter of fabricating structured cutting tools is further complicated by small dimensions of surface textures, the need to maintain proper dimension and shape tolerances and the fact that cutting tool materials are hard to process due to their high hardness and chemical properties (which is particularly problematic in case of tool materials used to machine difficult-to-cut alloys, such as PcBN).

The main goal of the following review is to discuss and investigate the application of laser processing to obtain cutting tool surface texturing basing on notable examples available in open literature and experimental research performed by the authors. The effects of using textured cutting tools on select aspects of cutting process performance (such as cutting forces, tool-workpiece interface friction and tool wear) are shown and discussed in the section Effects on cutting process performance as well.

\section{Texturing techniques and enhancement of process performance}

\section{Laser surface texturing of cutting tools}

Laser processing can be employed to produce surface structuring of cutting tools, as it allows for fabrication of structures with various shapes and geometries without the need for special tooling. Moreover, laser processing can be used regardless of material hardness, which is problematic when using mechanical processing methods, such as microscale machining or grinding. Still, laser processing equipment is very costly and an improper choice of parameters can result in reduced workpiece hardness, excessive heat-affected zone (HAZ) formation or poor surface quality/ insufficient repeatability of surface texturing patterns. Finally, laser processing is currently too time consuming for industrially relevant mass-scale production applications.

As laser processing is deemed to be the most promising technology for application of surface texturing on cutting tools, a plethora of research regarding laser

\footnotetext{
* MSc. PhD Krzysztof Jarosz, jarosz.krzysztof91@gmail.com - Rutgers University, Ney Jersey, USA
} 


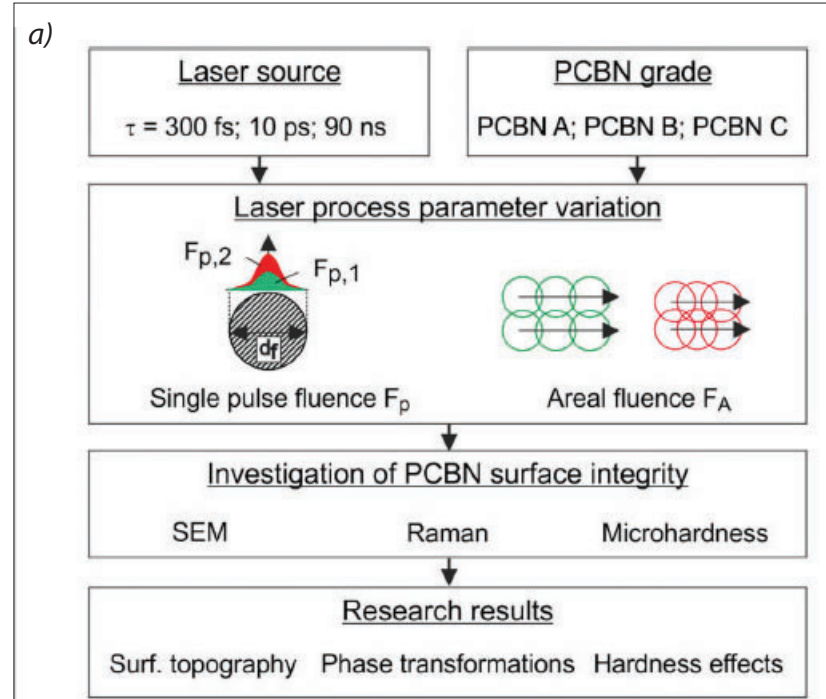

b)
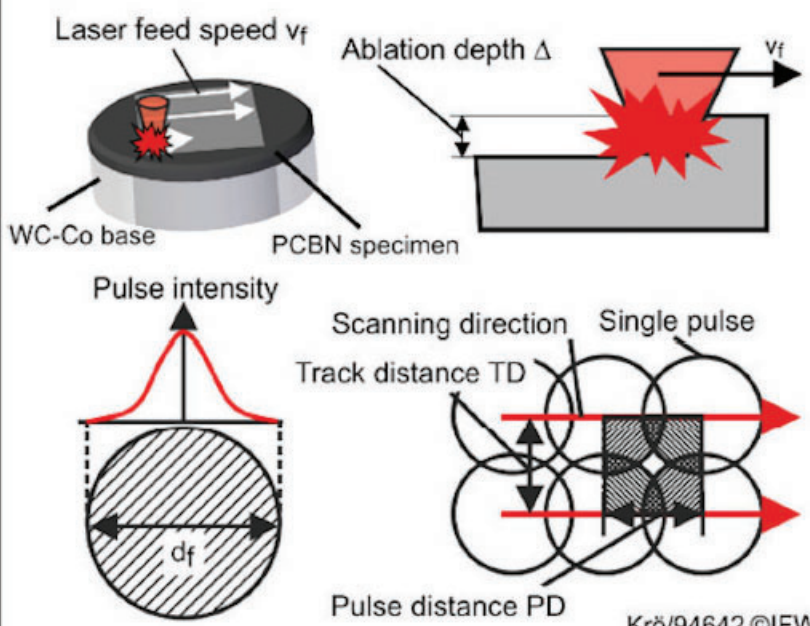

Fig. 2. Experiment flowchart (a) and investigated processing parameters $(b)$ in [4]

processing for surface texturing purposes is available in open literature.

The surface integrity of laser textured inserts for several different Polycrystalline cubic Boron Nitride (PcBN) content grades was investigated in [4]. Nanosecond-, picosecond- and femtosecond-pulsed lasers were employed in this comprehensive study, which focused mainly on insert microstructure and hardness after laser processing. Experiment outline and investigated parameters of the laser texturing process are shown in Fig. 2.

Presence of porous surfaces and surface cracks after laser processing was uncovered in the course of scanning electron microscopy (SEM) analysis. Vickers hardness measurements have shown that laser processing induced changes in insert microstructure and chemical composition can lead to a significant decrease in material hardness (up to $40 \div 50 \%$ in comparison with unprocessed PcBN) regardless of cBN content. Processing with the use of pico- and femtosecond pulsed lasers was shown to be less detrimental to insert hardness, as there is less melting and recrystallization. Moreover, the authors recommend using lower fluences for laser processing of PcBN inserts.
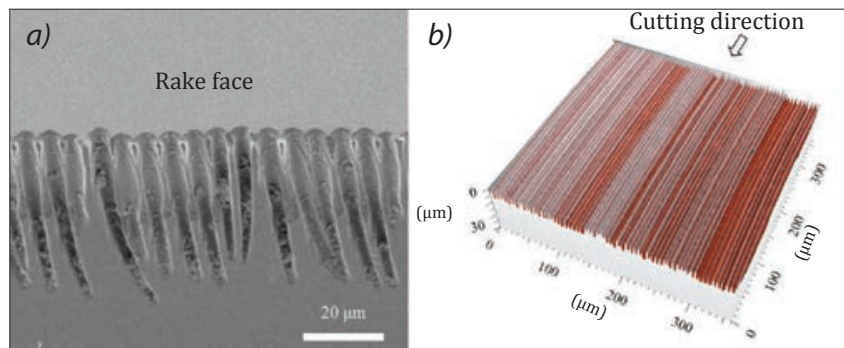

Fig. 3. SEM image of microgrooves fabricated by picosecond-pulsed laser on a diamond insert $(a)$ and workpiece surface topography after machining $(b)[7]$

The influence of laser processing parameters on generating laser surface texturing for high-friction applications was investigated in [5]. Although this research was focused on alloy and stainless steels (which are not used as modern cutting tool materials), it is still of relevance, as it shows that parameters of laser textured surfaces can be precisely controlled by properly adjusting pulse overlap, pulse energy and pulse frequency. Ta et al. [6] have investigated possibilities of generating hydrophobic surfaces using laser processing. Investigations were conducted for brass and copper alloys, which are not applicable as cutting tool materials due to their high plasticity, low melting/softening temperatures and low hardness. However, the results are promising and are likely to find application in cutting tool designs, as controlling surface wettability is relevant to more efficient utilization of cutting fluids.

An ultra-short (picosecond) pulsed laser to fabricate grooves on a single diamond tool, which is an exceptionally hard and brittle material, was successfully used in [7]. By appropriate choice of fluence and pulse frequency, the authors were able to control the process, producing grooves of desired width and geometry. The authors obtained hydrophobic workpiece surfaces when machining with textured tools, which shows a practical application of textured cutting tools in surface engineering. SEM image of textured diamond tool and resultant workpiece surface topography are shown in Fig. 3. As can be seen, this can have a possibly negative connotation, as the texture of the cutting tool is reflected by machined surface topography. Therefore, it can be inferred that certain surface texturing designs can be problematic to apply when a high-quality surface finish is desirable.

The effect of fluence, scanning speed and beam diameter on surface roughness, pattern repeatability and insert material hardness for laser surface texturing using a femtosecond pulsed laser to texturize uncoated carbide inserts was studied in [3]. The authors have found that using a higher fluence results in a better surface finish due to presence of a vaporization-dominant ablation regime. Using lower scanning speeds has resulted in less variation in groove geometry, but a higher heat-affected zone (HAZ) and decreased insert hardness were noted for scanning speeds above $10 \mathrm{~m} / \mathrm{s}$. Therefore, intermediate scanning speed values should be used to retain insert hardness, as it is crucial to insert durability and performance. 

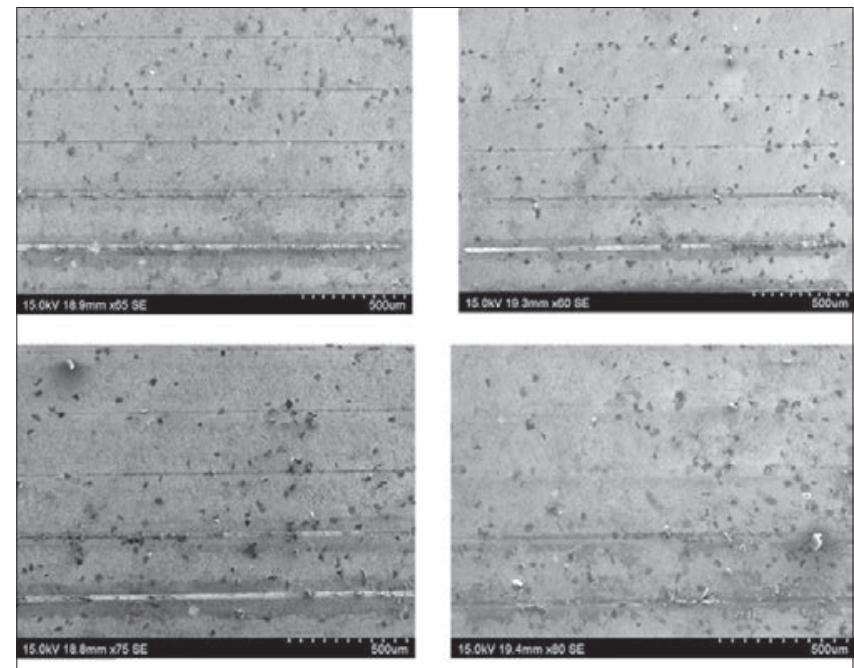

Fig. 4. Surface quality after fabrication with varying fluence and energy density levels [3]

The authors have also shown that very high fluence and low energy density is incapable of producing sufficient groove depth, whereas excessive spatter and low surface quality were noted when fluence was too low. This is shown in Fig. 4.

\section{Effects on cutting process performance}

An experimental case study for turning of AISI 4040 steel using uncoated carbide inserts, both unstructured and with rake face structing in the form of grooves applied on the rake face perpendicular to the main cutting edge was conducted in [3]. Lower cutting force values were noted for textured inserts. Textured insert used for testing and observed cutting force values are shown in Fig. 5.

The authors state that this is due to the grooves acting as cutting fluid reservoirs and improving chip flow (possibly due to reduction of chip-tool contact area) on the rake face of the tool. However, one has to note that tests with unstructured cutting tools were performed under dry cutting conditions - additional experiments for conventional and structured inserts conducted under lubricated cooling conditions would have produced more conclusive results.

In the recent work [2] the focus was on the performance of structured cutting inserts when machining Ti-6Al-4V grade titanium alloy under different cooling conditions. The authors start with an introductory remark that the main cause of tool failure in machining

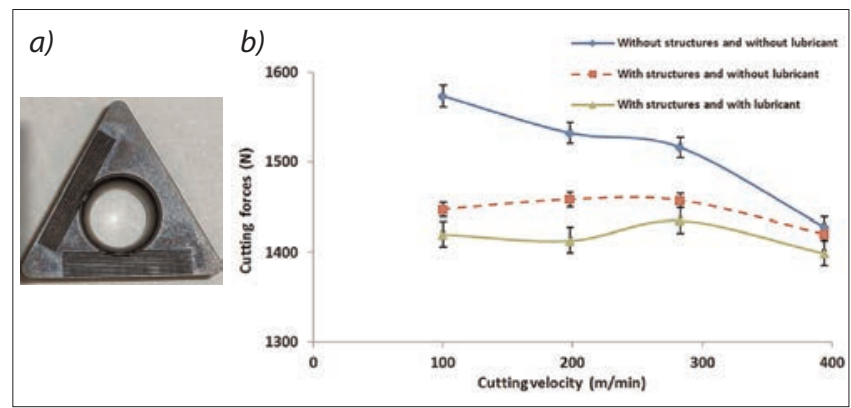

Fig. 5. Structured cutting insert ( $a$ ) and a comparison of cutting force values for conventional and structured inserts $(b)$ [3]

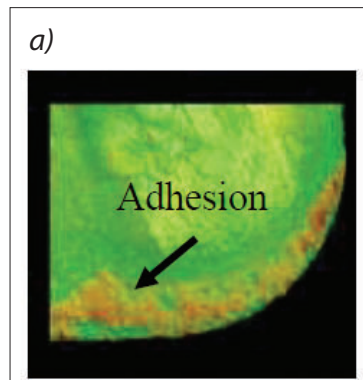

No texture

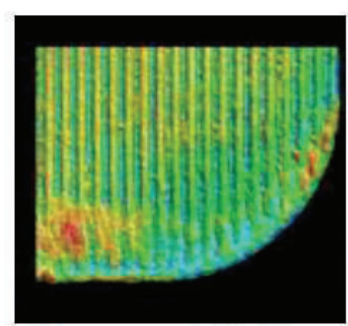

Concave width $50 \mu \mathrm{m}$

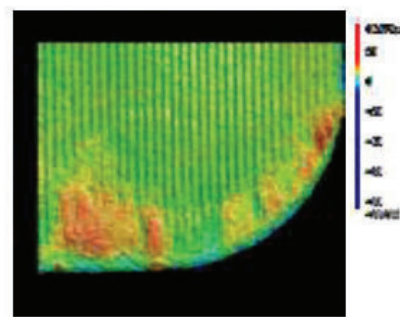

Concave width $20 \mu \mathrm{m}$

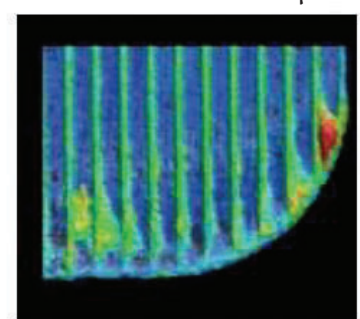

Concave width $100 \mu \mathrm{m}$ b)

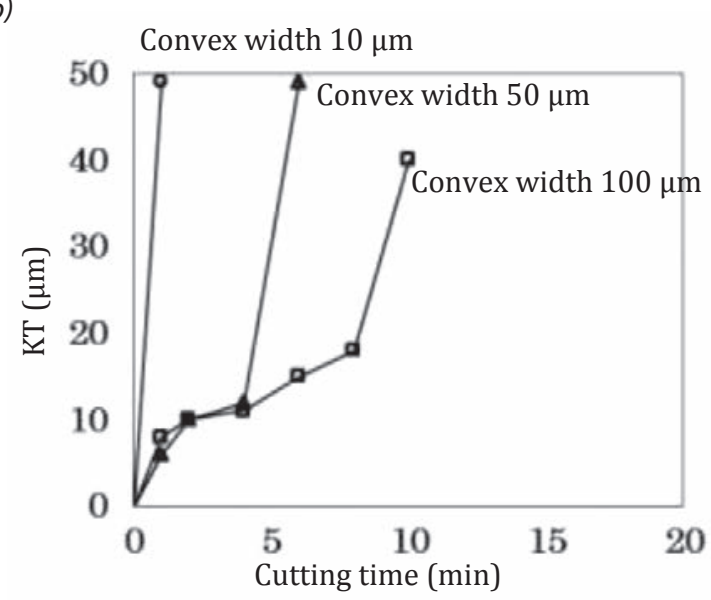

Fig. 6. Chip adhesion (a) and crater wear $(b)$ in turning of Ti-6Al-4V with laser textured carbide inserts [2]

Ti-6Al-4V is crater wear - therefore, it follows that the focus of presented study would be to minimize crater wear. The authors fabricated inserts with concave grooves parallel to the main cutting edge with three different groove widths $(20,50$ and $100 \mu \mathrm{m}$, respectively). Best results in terms of reducing chip adhesion and crater wear were noted for the highest groove width, as shown in Fig. 6.

Groove depth was also varied in the range of $10 \div 1000 \mu \mathrm{m}$. The authors have noted that shallow grooves $(10 \mu \mathrm{m})$ are easily blocked by the deformed chip - this behavior was observed when groove depth was equal to or greater than $20 \mu \mathrm{m}$. Longer tool life periods were noted for flood cooling conditions and high coolant application pressure - the beneficial effect of increased coolant supply and pressure is especially pronounced for textured cutting tools when compared with conventional tools. Overall best results in terms of mitigating tool wear were noted when using a combination of surface textured inserts and high-pressure flood coolant application.

The performance of three various textured insert designs in face turning of Inconel 718 nickel-based alloy, 


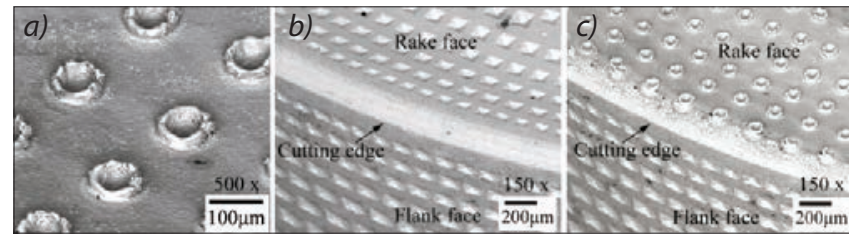

Fig. 7. Textured cutting inserts used in experimental investigations [1]

using cutting with conventional (untextured) inserts as a benchmark process for comparison purposes was investigated in [1]. Flank wear width $(V B)$, flank wear area $\left(V B_{\text {area }}\right)$, tool-chip contact length and surface topography of worn inserts were evaluated. Textures of investigated inserts are shown in Fig. 7.

Flank wear of varying width and area was observed for all inserts. Interestingly, no crater wear was observed after turning experiments. The authors concluded that employed surface textures had no significant effect on tool-chip contact area. This differs from textured tools with grooves, which are assumed to decrease the tool-chip contact area, as stated for example in the work of Fatima et al. [3]. Surface textures were found to be prone to being blocked with chip debris, which naturally reduces their effectiveness. Coolant burning and evaporation was also noted. Therefore, one can assume that the authors could explore possibilities of employing patterns of greater depth to aid coolant retention and mitigate blocking of texturings with chip debris. Best overall performance in terms of tool life (30\% improvement over conventional inserts) was noted for insert with dimples on the rake face and pyramidical pits on the flank face.

\section{Review summary}

The performed literature review investigates the subject of laser surface texturing of cutting tools for advanced manufacturing applications. Results from open literature were discussed in the following aspects:

- the effect of laser processing parameters on generated surface textures and

- the influence of surface textured tools on various aspects of cutting process performance.

The main takeaways from the literature reviews are as follows:

- Laser processing can be successfully applied to obtain surface structuring of cutting tools, the process can be controlled to obtain good pattern repeatability and quality. The detrimental effect of thermal processing on insert hardness and chemical composition can be mitigated, but is more pronounced in case of certain materials (for example PcBN).

- Proper selection of laser processing parameters is necessary to obtain the desired geometry, pattern accuracy and repeatability - research results shown in this review exhibit that an improper choice of parameters negatively affects surface texture quality (for example, ablation threshold will not be reached when pulse energy is too low, and excessive spatter will occur at high energy levels).
- Structured inserts show superior performance when compared with conventional (untextured) tool designs. Tool life and cooling effectiveness and can be improved by application of surface structuring. It can also help reduce chip-workpiece interface friction and cutting force values.

- Naturally, the preceding is true when texturing type and geometry is appropriate for a given application. Various designs are evaluated in open literature through means of experimental testing or predictive modeling.

- Industrially relevant application for mass-scale production necessitates speeding up the texture application process. Another limitation is the high cost of laser equipment. However, its versatility negates that drawback to a certain extent.

\section{REFERENCES}

[1] Alagan N.T., Zeman P., Hoier P., Beno T., Klement U. "Investigation of micro-textured cutting tools used for face turning of alloy 718 with high-pressure cooling". Journal of Manufacturing Processes. 37 (2019): 606-616, https://doi. org/10.1016/j.jmapro.2018.12.023.

[2] Sugihara T., Hasegawa M. "High-speed machining of Ti$6 \mathrm{Al}-4 \mathrm{~V}$ with a micro textured cutting tool focusing on coolant behaviors". LEMP 2020 Proceedings. Ohio, USA (2020).

[3] Fatima A., Whitehead D.J., Mativenga P.T. "Femtosecond laser surface structuring of carbide tooling for modifying contact phenomena". Proceedings of the Institution of Mechanical Engineers. Part B: Journal of Engineering Manufacture. 228, 11 (2014): 1325-1337, https://doi. org/10.1177/0954405413518516.

[4] Denkena B., Krödel A., Grove T. "Influence of pulsed laser ablation on the surface integrity of PCBN cutting tool materials". The International Journal of Advanced Manufacturing Technology. 101, 5-8 (2019) :1687-1698, https://doi. org/10.1007/s00170-018-3032-4.

[5] Dunn A., Carstensen J.V., Wlodarczyk K.L., Hansen E.B., Gabzdyl J., Harrison P.M., Hand D.P. "Nanosecond laser texturing for high friction applications". Optics and Lasers in Engineering. 62 (2014): 9-16, https://doi.org/10.1016/j. optlaseng.2014.05.003.

[6] Ta D.V., Dunn A., Wasley T.J., Kay R.W., Stringer J., Smith P.J., Shephard J.D. "Nanosecond laser textured superhydrophobic metallic surfaces and their chemical sensing applications". Applied Surface Science. 357 (2015): 248-254, https://doi.org/10.1016/j.apsusc.2015.09.027.

[7] Takayama N., Ishizuka J., Yan J. "Microgrooving of a singlecrystal diamond tool using a picosecond pulsed laser and some cutting tests". Precision Engineering. 53 (2018): 252-262, https://doi.org/10.1016/j.precisioneng.2018.04.009.

[8] Grzesik W. „Tribologiczne aspekty strukturyzowania powierzchni natarcia ostrza skrawającego" ("Tribological aspects of texturizing of the cutting tool's rake face"). Mechanik. 5-6 (2012): 418-423.

[9] Grzesik W. "Advanced machining processes of metallic materials". Amsterdam: Elsevier (2018), https://books.google.pl/books?hl=pl\&lr=\&id=j-_QA3u1D5EC\&oi=fnd\&pg=P $\mathrm{P} 1 \& \mathrm{dq}=$ Advanced + machining + processes $+o f+$ metallic $+\mathrm{m}$ aterials\&ots=ivdhDggbXR\&sig=Ytabh5rPo9aX6Hecmr0 ebLKqQc\&redir_esc $=y \# v=$ onepage $\& \mathrm{q}=$ Advanced $\% 20$ machining $\% 20$ processes $\% 20$ of $\% 20$ metallic $\% 20$ materials\&f=false. 